\title{
Best Practices for Artificial Intelligence in Life Sciences Research
}

Vladimir A. Makarov, PhD, MBA

Pistoia Alliance, 186 Lynn Oaks Ave, Thousand Oaks, CA 91320

vladimir.makarov@pistoiaalliance.org

Terry Stouch, PhD

Science for Solutions, 6211 Kaitlyn Ct, Princeton Junction, NJ 08550-5354

tstouch@gmail.com

Brandon Allgood, PhD

Integral, Inc., 1501 Mariposa Street \#426, San Francisco, CA 94107

allgood@integraltx.com

Chris D. Willis, PhD

Bristol-Myers Squibb Company, P.O. Box 4000, Princeton, NJ $08543-4000$

christopher.willis@bms.com

Nick Lynch, PhD

Curlew Research, Alderley Park, Macclesfield, Cheshire, SK10 4TG, UK nick.lynch@curlewresearch.com

Corresponding author: Makarov V.A. (vladimir.makarov@pistoiaalliance.org,phone +1-626222-5642)

Keywords: life sciences, research, drug discovery, artificial intelligence, machine learning

\section{Abstract:}

We describe 11 best practices for the successful use of Artificial Intelligence and Machine Learning in the pharmaceutical and biotechnology research, on the data, technology, and organizational management levels. 
Recently artificial intelligence and machine learning technologies in the biotechnology and pharmaceutical industries moved beyond experiments conducted by a few dedicated specialists and entered the industry mainstream. With this transition comes the need to formalize and publish the lessons learned by the early adopters. These lessons are not limited to the technology of artificial intelligence, but rather are in the best ways to apply Al methods in a business environment. In medicine, such lessons learned are already being recorded [1], however, these recommendations are extremely specific to the medical practice and not broadly applicable to drug discovery and biotech. This paper is the attempt to close this gap.

In the foregoing, we shall group the best practices into three categories: quality data, quality methods, and quality organization.

\section{Quality Data}

Data is the foundation of Al. It is becoming progressively easier and cheaper to produce and capture ever more data. Good mathematical models cannot be created using bad data. What's more, models based on data of inferior quality may not be immediately recognized as bad, leading to costly errors. Over the last two years we at the Pistoia Alliance AI and ML Center of Excellence conducted a broad survey of over 200 informatics professionals from the Pistoia Alliance member companies, and, consistently, access to quality data is identified as the top problem in $\mathrm{Al}$ and $\mathrm{ML}$ programs (70\% to $90 \%$ of respondents in different years). Work effort needed to cleanse and wrangle the raw data is identified as the main obstacle $(76 \%$ of respondents) to overcoming issues with data quality.

Best Practice 1. Do invest in domain knowledge needed to properly analyze the data in the highly specialized areas. Being able to understand the details in experiment set-up and study planning are essential skills not only for the bench scientists, but for the computational modelers as well. Be aware of the properties of the dataset one works with and of any inconsistencies that may invalidate the study.

In biology, some experiments that are considered simple by specialists may involve a lot of tacit details, making them in fact incredibly complex. Metadata used to capture facts related to data acquisition and related experimental information may be just as complex and diverse. Just a few examples: solubility and stability of the compounds, possible contaminants, variation in temperature and humidity during the experiments, sources of reagents and other materials, expiration dates. Instrument qualifications might be useful to confirm whether the particular instrument was or was not in need of repair when the experiment was performed. Even the source of distilled water can be of value. The degree of metadata utilization in biology varies with the maturity of the specific experimental techniques, and for the newest and hence the most interesting methods might be flat out insufficient. . On the other hand, for the wellestablished assay types (solubility, octanol-water partition coefficient, pKa, plasma protein binding, microsomal clearance) and for molecular structure determination the typical sources of error, [the] means to control them, and ways to detect errors in meta-analysis are extensively documented [2-11]. One should also be cognizant of the typical ranges of values and errors of the properties being measured. In many bioassays the random (aleatoric) error may be quite large in comparison to the typical data range $[3,5]$. 
Best Practice 2. Use only quality data for training of models. These data must be "fit for purpose". It is sensible to avoid data sets with insufficient information (metadata).

While it is hard to define perfection, describing what makes data really bad is easier. Lack of experimental context (metadata), incompleteness of data, confusion of units, problematic nomenclature, tautomerism, and misapplication of automated structure recognition tools are some commonly mentioned problems [9]. We should also add what is perhaps the most sinister issue: systemic error due to undocumented incompatibilities in experimental protocols. It happens frequently in drug discovery where it is found that the same measurements can vary substantially between different laboratories. Quality of data can also be in question when some of the data is accumulated over time and the definition of what's being measured might evolve during that time. Guides for curation of scientific data [2,9] and statistical tools for control of inter- and intra-site assay variability are available [11]. Meta-analysis based on literature sources or databases with little information about that data other than the particular data points themselves is risky. On the other hand, finding consistent data sets for metaanalysis studies is often hard, a problem that the authors of this paper witnessed first-hand on multiple occasions, and which is in shocking contrast with the popular claims of "data deluge". In literature reviews it has been determined that no more than $20 \%$ of published data may be viewed as truly reliable $[12,13]$. For model building the data uncertainty matters translate into recommendations for gradual build-up of models starting with training on high quality data, and if that is satisfactory, retraining the model using some of the potentially useful data, and for ensemble models [9]. In some cases, these activities may lead the user to the discovery of errors in the primary data (examples in ref. [9]).

Best Practice 3. Use a standard methodology such as FAIR for life cycle planning for scientific data.

Long-term data management plans should take into account the evolution of data and business needs for these data over time, forming a data life cycle. Significant investments are often made in talent and infrastructure for computational research, but investment towards data life cycle is just as often insufficient. Further, there is an emergent methodology for data management known as "FAIR principles" [14] that stands for "Findable, Accessible, Interoperable, and Reusable" and provides guidance for the best practices in scientific data management. Estimates of return on investment of FAIR implementation indicate a very large [15] opportunity cost of not using the FAIR principles for research data.

\section{Quality Models}

Good science must be reproducible, but model quality goes beyond the raw performance metrics.

Best Practice 4. Publish model code, and testing and training data, sufficient for reproduction of research work, along with model results. 
It should not be new to our readers that ML models ought to be validated on consistent but independent training and testing data sets. Cross-validation is a key part of model training, validation, and how test sets (hold outs) are used in the process to minimize the sampling bias of machine learning models. Data partitioning should be considered carefully and also reported in the description of the model. Despite the apparent obviousness of these points, the use case statistics show a troubling picture. For example, in a recent review of the diagnostic analysis of medical images with $\mathrm{Al}$, only in 31 (or 6\%) out of 516 papers published in the first half of 2018, the authors performed external validation, and none used best practices in clinical trial design [16]. We further recommend that publication of model results be accompanied by publication of appropriate data sets and model code, ready to be revalidated with minimal effort. Computational notebooks [17] are good tools for such communications; see also https://paperswithcode.com/. Models that fail in validation should be removed from production pipelines.

Best Practice 5. Use a model management system.

$\mathrm{Al}$ and ML models are not static, but tend to evolve over time. Therefore, just like software, infrastructure and data, mathematical models also have life cycles. Improper management of models may lead to regressive performance, massive model rebuilding efforts and silent failures by the models. These aspects, not a failure of the underlying modeling, often lead to the disillusionment seen in new technology adoption. In order to mitigate these problems, systems for model management, deployment, testing, versioning, meta-data capture and provenance need to be established. In software, this same concept is called continuous integration / continuous deployment $(\mathrm{Cl} / \mathrm{CD})$. While similar to software, model management constitutes a different type of technical debt, as is explained in [18]. Open-source systems for model versioning and provenance are currently emerging (MLflow (https://mlflow.org/), Sacred (https://github.com/IDSIA/sacred), DVC (https://dvc.org/)), and commercial vendors are also making offers in this space (SAS Model Manager). Policies and practices of test writing and running (as described in the previous section) are also required. This can be achieved using most $\mathrm{Cl} / \mathrm{CD}$ software systems with additional software harnesses. Testing should include change tests to ensure that models don't change due to external dependencies, such as, code versions and libraries versions, tests to ensure that a priori determined quality metrics are being met, such as, accuracy and degeneration in applicability domains, and tests that, at least for tracking reasons, ensure that the models are improving over time. Policies and practices for meta-data capture and provenance also need to be established. Model formats, such as, ONNX, allow for meta-data capture within the model. All of these aspects include both policy and practice backed by a set of emerging tools.

\section{Best Practice 6. Select ML methods that fit the specific use case or problem class.}

Not all problems can be equally well solved by all methods. For instance, deep learning works in images, text, sound, etc, because the subsets of possible features are very narrow in these applications but is less useful in unstructured data. Typical Neural Net technologies require a lot of data for training and therefore are not optimal when dealing with small data sets. A comparison of a multitude of ML techniques in medical applications with a discussion of their relative merits and regulatory matters may be found in $[19,20]$. The field of algorithm selection is an active research area in machine learning $[21,22]$. Regulatory compliance is becoming a key factor in ML method selection in life sciences as the ability to explain the model predictions 
is scrutinized by regulators [23]. Sensible advice is to use Occam's razor, when faced with a new problem. Experiment with different suitable ML technologies and feature sets starting with the simplest methods and features and then working toward more complex ones. In some cases, this process can be automated. This type of approach will also help to avoid overfitting.

\section{Quality Organization}

High profile, national headlines that attract the attention of executives frequently accompany $\mathrm{Al} / \mathrm{ML}$ advances in pharma/biotech as well as other industries. However, the press associated with cutting edge $\mathrm{Al} / \mathrm{ML}$ tends to focus on the success stories. The true effort needed to realize the disruptive power of this new technology, and the time lines from defining an Al/ML strategy through implementing a production grade solution rarely get the headlines. For an organization to realize the potential of $\mathrm{Al} / \mathrm{ML}$, the people component of an organization is equally as important as the data and models that drive the technology component.

Best Practice 7. Managers of commercial firms tend to expect miracles of new technologies (that they do not fully understand) on the up side of the technology curve. This leads to broken promises and bad financial decisions. As a practitioner, first educate one's colleagues what $\mathrm{Al} / \mathrm{ML}$ is and is not while setting the right expectations.

A typical pharma or biotech company can fall victim to two critical issues. On one hand, the C-suite executives realize the transformative power of the $\mathrm{Al} / \mathrm{ML}$, but may lack knowledge of technical details; while the technology managers' command of "under-the-hood" systems may come at the expense of the organizational vision. It is therefore critical for companies to form strategic partnerships with their Al technology where true collaborations result in mutual value, such as in the form of co-authorships (a recent example may be seen in [24]). On the other hand, the challenges with unstandardized, siloed data can further exacerbate the unrealistic expectations [25] of the scientific advances aiming to process the data, which as previously discussed, is not always reliable data. This has been true over multiple technology cycles in the past, from the classic bioinformatics approaches of the late 1980-s to the genomics revolution of the 2000-s, and, in our opinion, will remain so in the Al technology cycle as well.

Best Practice 8. Add the skill sets relevant to the $\mathrm{Al} / \mathrm{ML}$ to the company leadership.

Applying Al applications across R\&D and the enterprise [26] requires organizational change. As part of this change, there is a need for flexibility in adopting new business models and capabilities as well as a need for cross-domain leadership roles in management and science. In order to take advantage of the opportunities that digital transformations and in particular $\mathrm{Al} / \mathrm{ML}$ offer, new roles are commonly being added to pharma and biotech organizations. These new roles expand to the $\mathrm{C}$-suite level, as there is a multitude of $\mathrm{Al}$ applications across $\mathrm{R} \& \mathrm{D}$ as well as the enterprise [26]. This top down leadership approach is required to drive organizational change while supporting the adopting of new business models, processes and capabilities resulting from $\mathrm{Al} / \mathrm{ML}$ implementation and adoption. In particular, chief digital/data officers (CDOs) are tasked with identifying the areas $\mathrm{Al} / \mathrm{ML}$ can bring positive impact to business models, processes and capabilities resulting in a need to bring on board highly specialized talent [27]. Because CDOs work at the horizontal level of an organization, the Al 
projects they prioritize are those with the most overall business value versus being tied to functional domain. These prioritized projects are therefore most likely to be inherently disruptive while addressing a specific pain point whether its small molecule design in discovery or study design in clinical operations. This new role of the CDO expands on the traditional CIO type function focused on managing the IT landscape.

Best Practice 9. An advantageous business model is a combination of Al models and human decision-making, where mathematical models aid humans in generating datadriven insights but do not replace them.

In addition to defining the current state organizational transformation needs, a CDO's AI/ML roadmap should incorporate a vision for how the organization and technology interact in the future state. As discussed in Best Practice \#1, this vision needs to incorporate the right domain expertise to help elevate the overall organizational AI IQ. The current prevailing ideal that is directly relevant to pharma and biotech is a combination of $\mathrm{Al} / \mathrm{ML}$ technology aiding and working alongside human decision-makers [28]. In this ideal state, the $\mathrm{Al} / \mathrm{ML}$ algorithms and mathematical models aid humans but do not replace them. Humans are part of the creative process of identifying the right data, training and ensuring no bias exists in the models, and ultimately making decisions based on the model outputs. The role of $\mathrm{Al} / \mathrm{ML}$ technology is in using computational power to speed up processes and performing technical tasks beyond human capabilities. This "human plus machine" framework ultimately allows the scientists to focus on the high value, insight focused activities in a data-driven manner [29].

Best Practice 10. When implementing models take on an experimental mindset. Scale up fast and fail fast if needed. Accept that failure is part of the learning process. A bad model that is quickly deemed worthless is better than a deceptive model.

In addition to the need for organizational talent based on $\mathrm{Al} / \mathrm{ML}$ skillsets, organizations that want to truly embrace $\mathrm{Al} / \mathrm{ML}$ need to adopt and embrace an experimental mindset. Not unlike running an experiment in a lab, with $\mathrm{Al} / \mathrm{ML}$ it is important to generate hypotheses while developing the appropriate methods to test the hypotheses. Ultimately, failure in $\mathrm{Al} / \mathrm{ML}$ is part of the continuous learning process and "failing fast" is not an organizational set back. For those experiments that work, there should be a plan to expand and scale. Pharma and biotech drug discovery is in a unique industry position to be a leader in embracing an experimental mindset when it comes to $\mathrm{Al} / \mathrm{ML}$.

Best Practice 11. Creating an "Al Center of Excellence" is one way to focus on moonshot type innovation centered on solving multiple business driven use cases with $\mathrm{Al}$ at the same time.

Given the multitude of $\mathrm{Al} / \mathrm{ML}$ applications and opportunities across life sciences organizations at the enterprise level, it is important to have an organizational structure and governance that can centralize and standardize $\mathrm{Al} / \mathrm{ML}$ activities. There are a variety of names that embrace this organization structure and governance such as "Al Center of Excellence," "Digital Al Labs" or "Al Innovation Center" but in all cases they share the same goal. Ultimately, all AI/ML activities need to deliver value to the organization and will span from low hanging fruit from to moonshot type projects. While still an emerging technology, projects incorporating Al were mostly spearheaded within specialized functional groups and in collaboration with service 
providers with specific expertise. However now with the value proposition clear across the biopharma enterprise, expertise available in house and full support provided by the C-suite, the decentralized model is becoming less prominent. Now it is more common for external service providers to be brought in to increase the speed to complete and scale Al projects versus define and guide the technological journey. The need for centralization and standardization comes down to being able to manage and assess multiple projects across $R \& D$ at the same time. With most large pharma organizations, multiple use cases are ongoing at the same time within single value chain domains such as drug discovery research. This initial experimental mindset is important but ultimately before scaling to production, it is important to be able to assess common success criteria while quantifying the potential business value. Those organizations who are able to pinpoint and scale the highest value $\mathrm{Al} / \mathrm{ML}$ use cases will become leaders within the life sciences digital ecosystem.

In conclusion, there are a number of best practices that all organizations should strive to implement in order to achieve a positive return from investment in $\mathrm{Al} / \mathrm{ML}$. When these best practices are not followed the organization runs a high risk of undermining the potential and ultimately falling beyond competitors as we enter what is now being called the 4th Industrial Revolution [30]. 


\section{References:}

1. Vollmer S, Mateen BA, Bohner G, Király FJ, Ghani R, Jonsson P, et al. Machine learning and artificial intelligence research for patient benefit: 20 critical questions on transparency, replicability, ethics, and effectiveness BMJ 2020; doi:10.1136/bmj.I6927 (http://www.bmj.com)

2. Beck $B$, Seeliger $D$, Kriegl JM. The impact of data integrity on decision making in early lead discovery. J Comput Aided Mol Des 2015; 9; 911-21; doi: 10.1007/s10822015-9871-2.

3. Colclough N, Wenlock MC. Interpreting physicochemical experimental data sets. J Comput Aided Mol Des 2015; 9; 779-94; doi:10.1007/s10822-015-9850-7.

4. Deller MC, Rupp B. Models of protein-ligand crystal structures: trust, but verify. J Comput Aided Mol Des 2015; 9; 817-36; doi:10.1007/s10822-015-9833-8 .

5. Guthrie JP. Presenting data in such a fashion that they can be used by other scientists. J Comput Aided Mol Des 2015; 9; 837-46; doi:10.1007/s10822-015-9868$x$.

6. Krimmer SG, Klebe G. Thermodynamics of protein-ligand interactions as a reference for computational analysis: how to assess accuracy, reliability and relevance of experimental data. J Comput Aided Mol Des 2015; 9; 867-83; doi:10.1007/s10822015-9867-y.

7. Papadatos G, Gaulton A, Hersey A, Overington JP. Activity, assay and target data curation and quality in the ChEMBL database. J Comput Aided Mol Des. 2015; 9; 885-96; doi:10.1007/s10822-015-9860-5.

8. Segall MD, Champness EJ. The challenges of making decisions using uncertain data. J Comput Aided Mol Des. 2015; 9; 809-16. doi: 10.1007/s10822-015-9855-2.

9. Waldman M, Fraczkiewicz R, Clark RD. Tales from the war on error: the art and science of curating QSAR data. J Comput Aided Mol Des. 2015; 9; 897-910; doi:10.1007/s10822-015-9865-0.

10. Wätzig H, Oltmann-Norden I, Steinicke F, Alhazmi HA, Nachbar M, El-Hady DA, et al. Data quality in drug discovery: the role of analytical performance in ligand binding assays. J Comput Aided Mol Des. 2015; 9; 847-65; doi:10.1007/s10822-015-9851-6.

11. Winiwarter S, Middleton B, Jones B, Courtney P, Lindmark B, Page KM, et al. Time dependent analysis of assay comparability: a novel approach to understand intraand inter-site variability over time. J Comput Aided Mol Des. 2015; 9; 795-807; doi:10.1007/s10822-015-9836-5.

12. (was \#3) Stouch T. The sociology of pharmaceutical research: Challenges in the interpretation, understanding, and use of Drug Discovery data. 241st National Meeting of the American Chemical Society 2011.

13. Brown S, Muchmore SW, Hajduk PJ. Healthy skepticism: assessing realistic model performance. Drug Discov Today 2009; 14; 420-7.

14. Wise J, Grebe de Barron A, Splendiani A, Balali-Mood B, Vasant D, Little E, et al. Implementation and relevance of FAIR data principles in biopharmaceutical $R \& D$, Drug Discov Today 2019; 24; 933-8.

15. Directorate-General for Research and Innovation, PwC EU Services. Cost of not having FAIR research data. European Commission 2018; doi:10.2777/02999 (https://publications.europa.eu/en/publication-detail/-/publication/d375368c-1a0a11e9-8d04-01aa75ed71a1/language-en) 
16. Kim DW, Jang HY, Kim KW, Shin Y, Park SH. Design Characteristics of Studies Reporting the Performance of Artificial Intelligence Algorithms for Diagnostic Analysis of Medical Images: Results from Recently Published Papers. Korean J Radiol 2019; 20; 405-10.

17. Rule A, Birmingham A, Zuniga C, Altintas I, Huang SC, Knight R, et al. Ten simple rules for writing and sharing computational analyses in Jupyter Notebooks. PLOS Comput Biol 2019; doi:10.1371/journal.pcbi.1007007 (https://journals.plos.org/ploscompbiol/).

18. Sculley D, Holt G, Golovin D, Davydov E, Phillips T, Ebner D, et al. Hidden Technical Debt in Machine Learning Systems. Advances in Neural Information Processing Systems 2015; 28; 2503-11

19. Forghani R, Savadjiev P, Chatterjee A, Muthukrishnan N, Reinhold C, Forghani B. Radiomics and Artificial Intelligence for Biomarker and Prediction Model Development in Oncology. Comput Struct Biotechnol J 2019; 1; 995-1008.

20. Miller DD. The medical Al insurgency: what physicians must know about data to practice with intelligent machines. NPJ Digit Med 2019; doi:10.1038/s41746-0190138-5 (https://www.nature.com/) .

21. Ali S, Smith KA. On learning algorithm selection for classification. Applied Soft Computing 2006; 6; 119-38.

22. Kotthoff LA. Preliminary Evaluation of Machine Learning in Algorithm Selection for Search Problems. AAAI Publications, Fourth Annual Symposium on Combinatorial Search 2011.

23. Food and Drug Administration (FDA). Proposed Regulatory Framework for Modifications to Artificial Intelligence/Machine Learning (AI/ML)-Based Software as a Medical Device, https://www.regulations.gov/document?D=FDA-2019-N-1185-0001; 2019 [accessed April $5^{\text {th }}$ 2020].

24. Szabo PM, Lee G, Ely S, Baxi V, Pokkalla $H$, Elliott $H$, et al. CD8+ T cells in tumor parenchyma and stroma by image analysis (IA) and gene expression profiling (GEP): Potential biomarkers for immuno-oncology (I-O) therapy. J Clin Oncol 2019; 37 (suppl; abstract 2594); doi: 10.1200/JCO.2019.37.15_suppl.2594 (https://meetinglibrary.asco.org/record/172738/abstract).

25. Schneider G. Automating drug discovery. Nat Rev Drug Discov 2017; 17; 97-113.

26. Vamathevan J, Clark D, Czodrowski P, Dunham I, Ferran E, Lee G, et al. Applications of machine learning in drug discovery and development. Nat Rev Drug Discov. 2019; 18; 463-77.

27. Fleming N. How artificial intelligence is changing drug discovery. Nature 2018; 557; doi:10.1038/d41586-018-05267-x (http://www.nature.com).

28. Green DVS, Pickett S, Luscombe C, Senger S, Marcus D, Meslamani J, et al. BRADSHAW: a system for automated molecular design. J Comput Aided Mol Des. 2020; 34; 747-65. doi:10.1007/s10822-019-00234-8 (https://link.springer.com/)

29. Daugherty PR, Wilson HJ. Human + Machine: Reimagining Work in the Age of Al. Brighton: Harvard Business Review Press; 2018.

30. Skilton M, Hovsepian F. The 4th Industrial Revolution: Responding to the Impact of Artificial Intelligence on Business. London UK: Palgrave Macmillan; 2017. 